\title{
类钙钛矿化合物 $(\mathrm{Ca} / \mathrm{Sr})_{2} \mathrm{CuO}_{2} \mathrm{Cl}_{2}$ 高压结构稳定性和 电性质
}

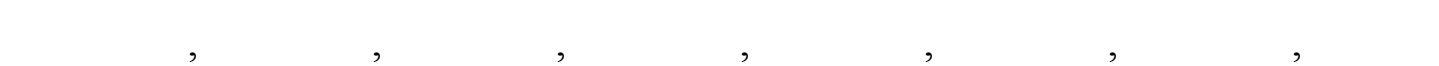

(1) 北京大学物理学院, 北京 100871 ;

(2) 中国科学院物理研究所, 北京 100080 ;

(3) 中国科学院高能物理研究所, 北京 100049

2007-12-27 收稿, 2008-03-06 接受

国家重点基础研究发展计划(编号: 2005CB724402)和国家自然科学基金(批准号: 50401009, 50701048)资助项目

摘要 利用金刚石压砧技术在 $0 \sim 31 \mathrm{GPa}$ 的压力范围内研究了常温下 $\mathrm{K}_{2} \mathrm{NiF}_{4}$ 型结构的 $(\mathrm{Ca} / \mathrm{Sr})_{2} \mathrm{CuO}_{2} \mathrm{Cl}_{2}$ 化合物的结构稳定性和电性质. 结果表明, $(\mathrm{Ca} / \mathrm{Sr})_{2} \mathrm{CuO}_{2} \mathrm{Cl}_{2}$ 化合物的晶格 结构在高压下是稳定的, 并且具有较大的各相异性压缩, 得到了 $\left(\mathrm{Ca} / \mathrm{Sr}_{2} \mathrm{CuO}_{2} \mathrm{Cl}_{2}\right.$ 化合物 的状态方程和体弹模量. 电学性质测量表明, 高压下 $\mathrm{Sr}_{2} \mathrm{CuO}_{2} \mathrm{Cl}_{2}$ 样品可能有电子结构相 关键词

高压 类钙钛矿型化合物 同步辐射 变发生.

高温超导体研究是凝聚态物质科学的重要前沿 领域. 高温超导体在晶体结构上属衍生的钙钛矿类 铜氧化物, 而压力非常适合稳定高度致密的钙钛矿 物质. 压力在高温超导体的研究中发挥着及其重要 的作用, 是研制新型高温超导材料的重要手段. 近年 来, 卤铜氧化合物作为理想的二维模型体系材料受 到人们的广泛关注, 在晶体结构上, 它由 $\mathrm{CuO}_{2}$ 平面 导电层和岩盐型的卤化物交替叠加而成. 与全氧化 物超导体相比较, 卤素原子的半径比氧原子大, 所以 结构中 $c$ 轴较长(如图 1 所示), 导致 $\mathrm{CuO}_{2}$ 平面导电层 与电荷库层的耦合减弱, 形成具有准二维的 $\mathrm{CuO}_{2}$ 平 面，是角分辨光电子谱(ARPES)和扫描隧道谱(STM)

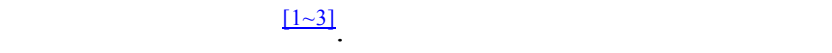
重要前驱体材料，例如，Jin等人 [4 8]利用“顶角氧掺 杂”机制，用 $(\mathrm{Ca} / \mathrm{Sr})_{2} \mathrm{CuO}_{2} \mathrm{Cl}_{2}$ 化合物作前驱体，高温 高压合成了卤铜氧化合物超导体. 因此, 开展高压下 $(\mathrm{Ca} / \mathrm{Sr})_{2} \mathrm{CuO}_{2} \mathrm{Cl}_{2}$ 化合物的结构性研究, 对探索高温超 导机制具有积极意义.

\section{1 实验}

利用固态反应法, 将纯度大于 $99.9 \%$ 的 $\mathrm{SrCO}_{3}$ 和 $\mathrm{CuO}$ 粉末以 $1: 1$ 的摩尔比混合并研磨均匀, 然后在

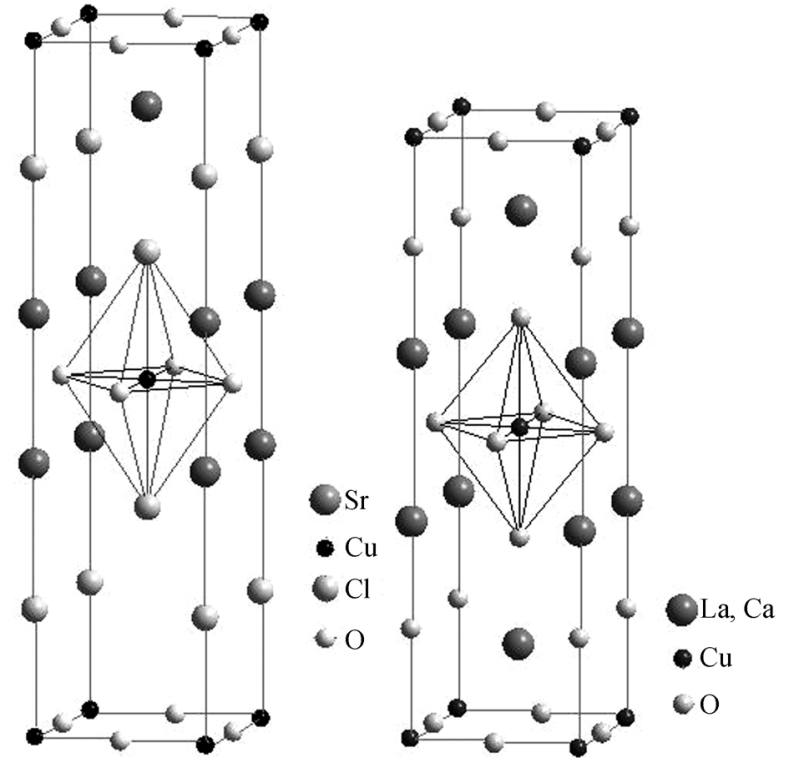

图 $1(\mathrm{Ca} / \mathrm{Sr})_{2} \mathrm{CuO}_{2} \mathrm{Cl}_{2}$ 和 $\mathrm{La}_{2} \mathrm{CuO}_{4}$ 的晶格结构示意图

$930^{\circ} \mathrm{C}$ 的条件下烧结, 保温 $24 \mathrm{~h}$, 并重复上述烧结、保 温过程, 即可得到单相的 $\mathrm{SrCuO}_{2}$, 然后将上述 $\mathrm{SrCuO}_{2}$ 与纯度大于 $99.9 \%$ 的 $\mathrm{SrCl}_{2}$ 以 $1: 1$ 摩尔比混合、 研磨均匀, 在 $950^{\circ} \mathrm{C}$ 的条件下烧结, 保温 $24 \mathrm{~h}$, 再重复 上述烧结、保温过程, 即可得到单相的 $\mathrm{Sr}_{2} \mathrm{CuO}_{2} \mathrm{Cl}_{2}$ 化合 
物. $\mathrm{Ca}_{2} \mathrm{CuO}_{2} \mathrm{Cl}_{2}$ 前驱体的制备是将 $99.9 \%$ 高纯的 $\mathrm{CaO}$ 和 $\mathrm{CuCl}_{2}$ 按 2:1 的摩尔比混合、研磨, 然后在 $800^{\circ} \mathrm{C}$ 的条 件下烧结, 保温 $12 \mathrm{~h}$ (重复烧结两次), 可得到单相的 $\mathrm{Ca}_{2} \mathrm{CuO}_{2} \mathrm{Cl}_{2}$ 化合物.

利用金刚石压砧实验技术，在不同范围的压力 下分别对 $(\mathrm{Ca} / \mathrm{Sr})_{2} \mathrm{CuO}_{2} \mathrm{Cl}_{2}$ 样品的晶体结构、电学性质 进行研究. 实验中采用金刚石高压装置, 金刚石压砧 台面直径为 $500 \mu \mathrm{m}$. 高压同步辐射实验是在北京同 步辐射多色 X 射线源上完成的, Pt 作为压力内标. 高 压电学性质测试采用准四引线方法进行.

\section{2 结果与讨论}

图 2 为常压合成的 $(\mathrm{Ca} / \mathrm{Sr})_{2} \mathrm{CuO}_{2} \mathrm{Cl}_{2}$ 样品的 $\mathrm{X}$ 射线 衍射图谱. $X$ 射线结构分析表明样品为单相, 具有四方

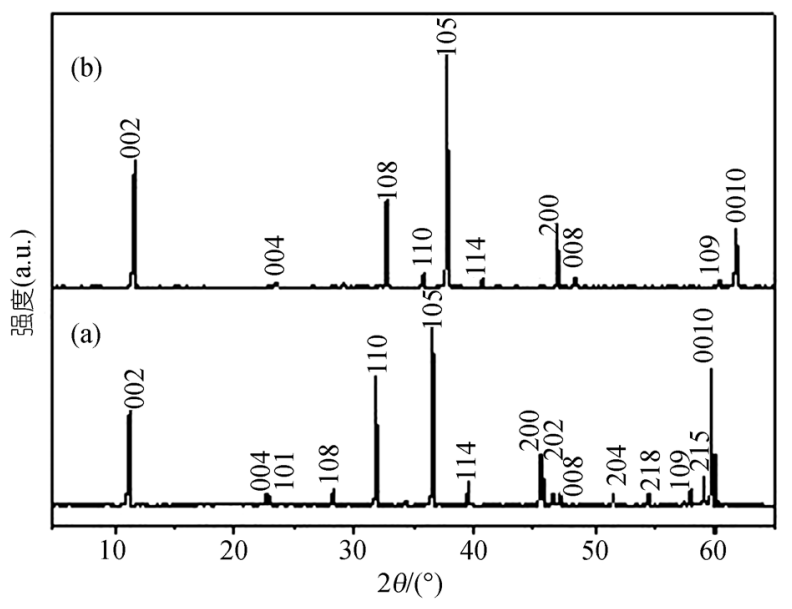

图 2 常压合成的 $(\mathrm{Ca} / \mathrm{Sr})_{2} \mathrm{CuO}_{2} \mathrm{Cl}_{2}$ 的 $\mathrm{X}$ 射线衍射图

(a) $\mathrm{Sr}_{2} \mathrm{CuO}_{2} \mathrm{Cl}_{2}$; (b) $\mathrm{Ca}_{2} \mathrm{CuO}_{2} \mathrm{Cl}_{2}$

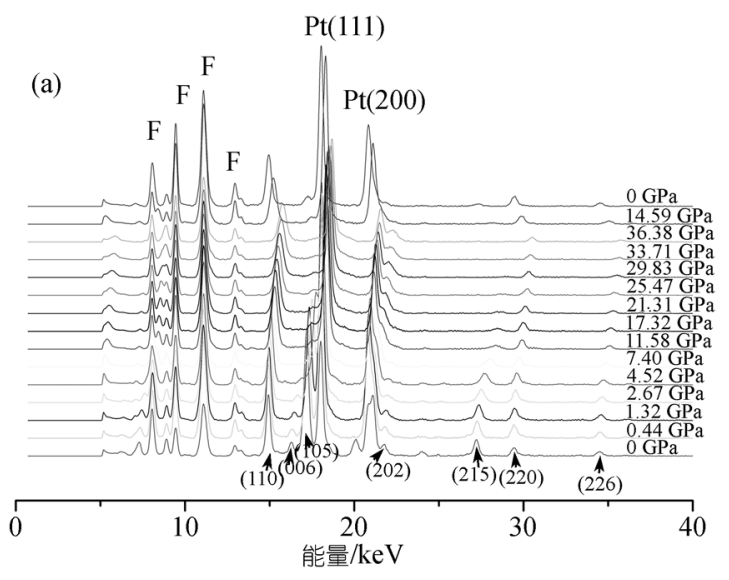

结构, 空间群为 $I 4 / m m m, \mathrm{Sr}_{2} \mathrm{CuO}_{2} \mathrm{Cl}_{2}$ 和 $\mathrm{Ca}_{2} \mathrm{CuO}_{2} \mathrm{Cl}_{2}$ 的晶格参数分别为 $a=3.9728(2) \AA, c=15.6206(4) \AA$ 和 $a=3.8659(1) \AA, c=15.0428(2) \AA$.

采用金刚石压砧技术，在 $0 \sim 30 \mathrm{GPa}$ 的压力范围 内对 $(\mathrm{Ca} / \mathrm{Sr})_{2} \mathrm{CuO}_{2} \mathrm{Cl}_{2}$ 样品的晶体结构和压缩性进行研 究. 高压能散 X 射线衍射结果如图 3 所示. 在衍射谱的 低能端出现一些来自样品和 Pt 标压材料的苂光峰. 从 图 3 中可以看出, 随着压力增加, 除了 $(\mathrm{Ca} / \mathrm{Sr})_{2} \mathrm{CuO}_{2} \mathrm{Cl}_{2}$ 样品的(110)最强峰的强度没有明显的变化以外, 其 他的样品峰强度明显地减弱. 当压力释放到常压时, 所有的样品峰又回到原来位置, 并且没有新峰出现, 这说明在实验压力范围内, $(\mathrm{Ca} / \mathrm{Sr})_{2} \mathrm{CuO}_{2} \mathrm{Cl}_{2}$ 的晶体结 构是稳定的. 根据图 3 所标的样品衍射峰, 使用最小 二乘法程序计算得到其晶格参数.

图 4 所示为晶格参数 $a$ 和 $c$ 的变化率随压力的变 化关系. 随着压力的增加, $a$ 和 $c$ 缓慢地减小. $a$ 轴的 减小可能是由 $\mathrm{Cu}-\mathrm{O}$ 键长的压缩引起的, 而 $c$ 轴的减 小可能是由 $(\mathrm{Ca} / \mathrm{Sr})_{2} \mathrm{Cl}_{2}$ 岩盐层的压缩引起的. $c$ 轴的压 缩率比 $a$ 轴大, 这说明在高压下此类化合物的压缩系 数具有较强的各向异性. 图 5 给出了 $(\mathrm{Ca} / \mathrm{Sr})_{2} \mathrm{CuO}_{2} \mathrm{Cl}_{2}$ 样品的体积压缩率随压力的变化关系. 根据压力和 压缩率数据, 利用 Birch-murnaghan 状态方程

$$
\begin{aligned}
P(\mathrm{GPa})= & \frac{3}{2} \times B_{0} \times\left[\left(\frac{V_{0}}{V}\right)^{\frac{7}{3}}-\left(\frac{V_{0}}{V}\right)^{\frac{5}{3}}\right] \\
& \times\left\{1-\left(3-\frac{3}{4} \times B_{0}^{\prime}\right) \times\left[\left(\frac{V_{0}}{V}\right)^{\frac{2}{3}}-1\right]\right\}
\end{aligned}
$$

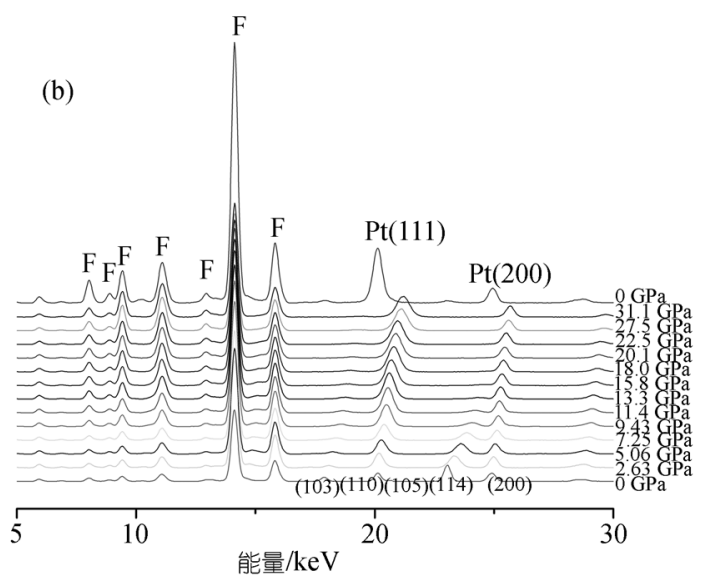

图 $3(\mathrm{Ca} / \mathrm{Sr})_{2} \mathrm{CuO}_{2} \mathrm{Cl}_{2}$ 样品的高压 $\mathrm{X}$ 射线能散谱

(a) $\mathrm{Ca}_{2} \mathrm{CuO}_{2} \mathrm{Cl}_{2}$; (b) $\mathrm{Sr}_{2} \mathrm{CuO}_{2} \mathrm{Cl}_{2}$ 

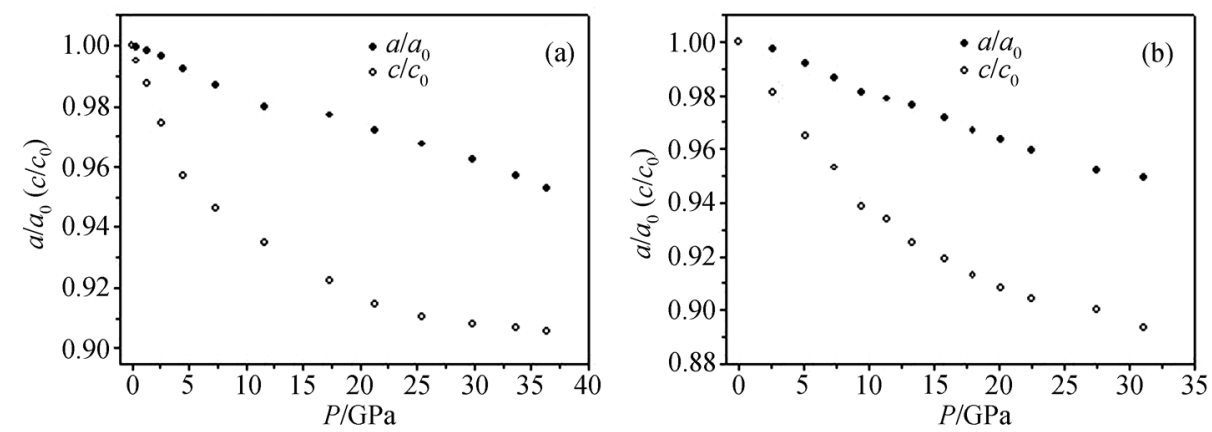

图 $4(\mathrm{Ca} / \mathrm{Sr})_{2} \mathrm{CuO}_{2} \mathrm{Cl}_{2}$ 样品的晶格参数 $a$ 和 $c$ 随压力的变化关系

(a) $\mathrm{Ca}_{2} \mathrm{CuO}_{2} \mathrm{Cl}_{2}$; (b) $\mathrm{Sr}_{2} \mathrm{CuO}_{2} \mathrm{Cl}_{2}$

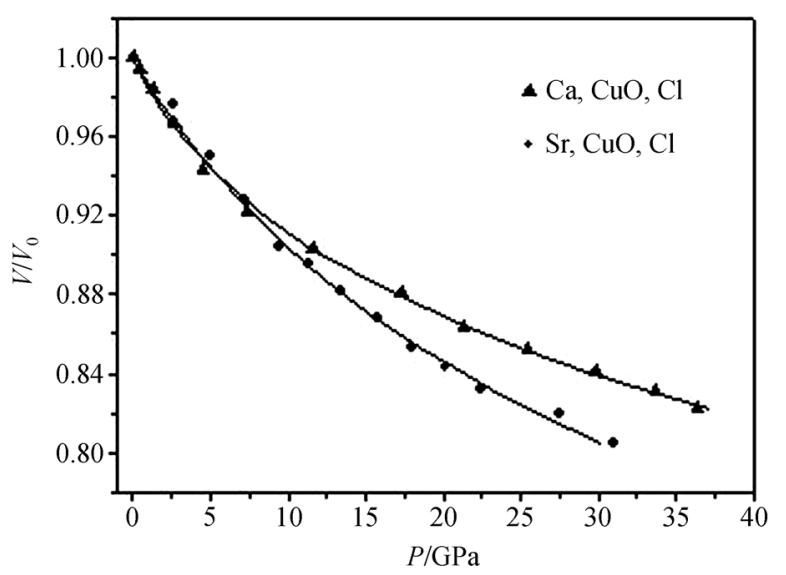

图 $5(\mathrm{Ca} / \mathrm{Sr})_{2} \mathrm{CuO}_{2} \mathrm{Cl}_{2}$ 样品的体积压缩率与压力的关系

拟合实验数据得到, 压力导数 $B_{0}^{\prime}=4, \mathrm{Ca}_{2} \mathrm{CuO}_{2} \mathrm{Cl}_{2}$ 和 $\mathrm{Sr}_{2} \mathrm{CuO}_{2} \mathrm{Cl}_{2}$ 的零压体弹模量分别为 $B_{0}=(116.8 \pm 3.8)$ $\mathrm{GPa}$ 和 $B_{0}=(86.5 \pm 1.3) \mathrm{GPa}$.

图 6 给出了 $\mathrm{A}_{2} \mathrm{CuO}_{2} \mathrm{Cl}_{2}(\mathrm{~A}=\mathrm{Ca}, \mathrm{Sr})$ 的电阻随压力的 变化关系, 可以看出在 $0 \sim 20 \mathrm{GPa}$ 的压力范围内, 两 个样品的电阻随压力的增加表现出不同的变化.
高压能散X射线衍射结果表明，所有样品的晶体结构 都是稳定的, 因此高压下晶格参数的变化可能会引 起能带结构的变化, 从而导致电阻的变化. 从图 6(a) 可以看到, 随着压力的增加, $\mathrm{Ca}_{2} \mathrm{CuO}_{2} \mathrm{Cl}_{2}$ 电阻值明显 减小, 但没有突变发生, 电阻值的减小可能是由于晶 粒与晶粒之间的紧密接触引起的, 因此没有电子结 构的相变发生. 图 6(b) 所示为 $\mathrm{Sr}_{2} \mathrm{CuO}_{2} \mathrm{Cl}_{2}$ 样品的电阻 随压力的变化关系, 在 $2 \mathrm{GPa}$ 时电阻突然下降, 然后 缓慢上升, 当压力达到 $13 \mathrm{GPa}$ 时, 电阻逐渐减小, 我 们认为这可能是由于该样品电子结构的变化所致, 但是, 本质的物理性质需要进一步的研究.

\section{3 结论}

利用 DAC 技术在 $0 \sim 30 \mathrm{GPa}$ 压力范围内对 $(\mathrm{Ca} / \mathrm{Sr})_{2} \mathrm{CuO}_{2} \mathrm{Cl}_{2}$ 超导体母体相化合物进行了原位高 压能散X射线衍射和电学性质研究. 高压同步辐射结 果表明, 在整个实验压力范围内 $(\mathrm{Ca} / \mathrm{Sr})_{2} \mathrm{CuO}_{2} \mathrm{Cl}_{2}$ 样品 的晶体结构是稳定的, 没有结构相变发生. 根据体积 压缩率与压力的关系, 利用 Birch-Murnaghan 状态方 程拟合实验数据, 得到 $\mathrm{Ca}_{2} \mathrm{CuO}_{2} \mathrm{Cl}_{2}$ 和 $\mathrm{Sr}_{2} \mathrm{CuO}_{2} \mathrm{Cl}_{2}$
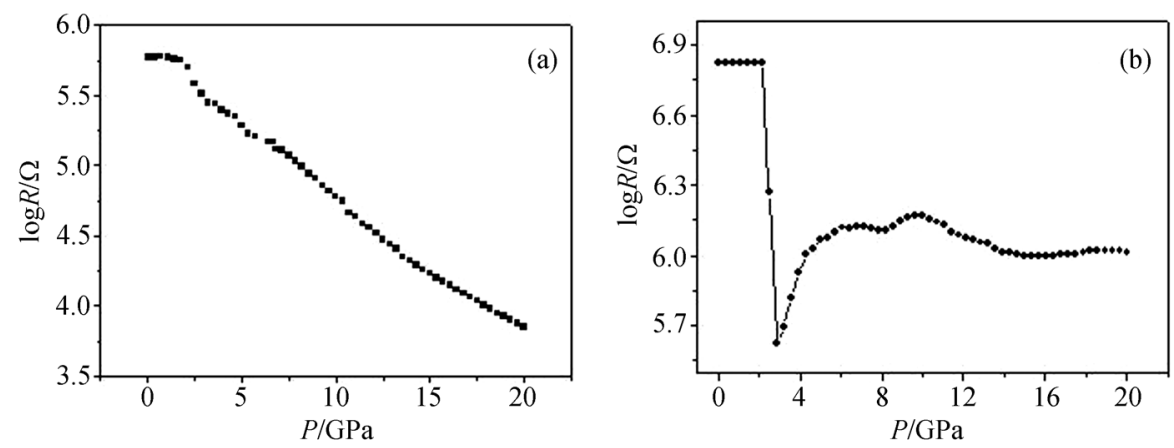

图 $6(\mathrm{Ca} / \mathrm{Sr})_{2} \mathrm{CuO}_{2} \mathrm{Cl}_{2}$ 样品的电阻随压力的变化关系

(a) $\mathrm{Ca}_{2} \mathrm{CuO}_{2} \mathrm{Cl}_{2}$; (b) $\mathrm{Sr}_{2} \mathrm{CuO}_{2} \mathrm{Cl}_{2}$ 
的零压体弹模量分别为 $(116.8 \pm 3.8) \mathrm{GPa}$ 和 $(86.5 \pm 1.3)$

$\mathrm{GPa}$ (压力导数 $B_{0}{ }^{\prime}=4$ ). 在 $0 \sim 20 \mathrm{GPa}$ 压力下对 $\mathrm{Ca}_{2} \mathrm{CuO}_{2} \mathrm{Cl}_{2}$ 和 $\mathrm{Sr}_{2} \mathrm{CuO}_{2} \mathrm{Cl}_{2}$ 原位高压电性质测量结果
发现, $\mathrm{Ca}_{2} \mathrm{CuO}_{2} \mathrm{Cl}_{2}$ 和 $\mathrm{Sr}_{2} \mathrm{CuO}_{2} \mathrm{Cl}_{2}$ 的电阻随压力的增加 表现出明显不同的变化关系. 研究认为高压下 $\mathrm{Sr}_{2} \mathrm{CuO}_{2} \mathrm{Cl}_{2}$ 样品可能有电子结构相变发生.

\section{参考文献}

1 Ronning F, Kim C, Feng D L, et al. Synthesis, structure, and properties of $\mathrm{Sr}_{2} \mathrm{CuO}_{2} \mathrm{Cl}_{2}$. Phys Rev B, 1990, 41: 1921-1925 [DOI]

2 Hanaguri T, Lupien C, Kohsaka Y, et al. A 'checkerboard' electronic crystal state in lightly hole-doped $\mathrm{Ca}_{2-} \mathrm{Na}_{x} \mathrm{CuO}_{2} \mathrm{Cl}_{2} \cdot \mathrm{Nature}$, 2004, 430: 1001-1005[DOI]

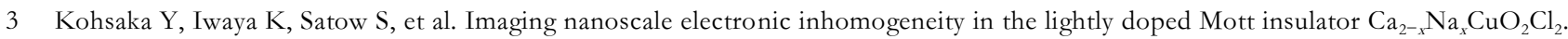
Phys Rev Lett, 2004, 93: 097004 [DOI]

4 Jin C Q, Wu X J, Laffez P, et al. Superconductivity at $80 \mathrm{~K}$ in $(\mathrm{Sr}, \mathrm{Ca})_{3} \mathrm{Cu}_{2} \mathrm{O}_{4+\delta} \mathrm{Cl}_{2-y}$ induced by apical oxygen doping. Nature, 1995, 375: $301-303 \underline{\text { [DOI] }}$

5 Jin $\mathrm{C}$ Q, Wu X J, Adachi S, et al. High-pressure synthesis and superconducting properties of the oxychloride superconductor $(\mathrm{Sr}, \mathrm{Ca})_{3} \mathrm{Cu}_{2} \mathrm{O}_{4+\delta} \mathrm{Cl}_{2-y}$. Phys Rev B, 2000, 61: 778-783 $\underline{\text { [DOI] }}$

6 Jin C Q. High pressure synthesis of novel high $T_{\mathrm{c}}$ superconductors. High Pressure Res, 2004, 24: 399-408 [DOI]

7 Haffner S, Brammeier D M, Olson C G, et al. No well-defined remnant Fermi surface in $\mathrm{Sr}_{2} \mathrm{CuO}_{2} \mathrm{Cl}_{2}$. Phys $\mathrm{Rev}_{\mathrm{B}} \mathrm{B}, 2001,63$ : 212501[DOI]

8 Liu Q Q, Qin X M, Yu Y, et al. High pressure synthesis of a new superconductor $\mathrm{Sr}_{2} \mathrm{CuO}_{2+\delta} \mathrm{Cl}_{2-y}$ induced by apical oxygen doping. Physica C, 2005, 420: 23-29 $\underline{\underline{[D O I}]}$ 